\title{
Traduire
}

Revue française de la traduction

$230 \mid 2014$

À la croisée du texte et de l'image

\section{Les traducteurs au cinéma}

\section{Graham macLachlan}

\section{OpenEdition}

\section{Journals}

Édition électronique

URL : http://journals.openedition.org/traduire/625

DOI : 10.4000/traduire.625

ISSN : 2272-9992

\section{Éditeur}

Société française des traducteurs

\section{Édition imprimée}

Date de publication : 15 juin 2014

Pagination : 63-69

ISSN : 0395-773X

\section{Référence électronique}

Graham macLachlan, «Les traducteurs au cinéma », Traduire [En ligne], 230 | 2014, mis en ligne le 15 juin 2016, consulté le 30 avril 2019. URL : http://journals.openedition.org/traduire/625 ; DOI : 10.4000/ traduire.625 


\section{Les traducteurs au cinéma}

\section{Graham macLachlan}

Le portrait que le cinéma dresse du traducteur et de la traduction(1) n'est sans doute pas sans conséquence sur la manière dont le public perçoit la profession, surtout quand le message est répété à l'infini. Citons à ce propos l'exemple de Lost in Translation, film de Sofia Coppola sorti en 2003, dont le titre est repris par défaut dans la presse anglophone pour tout article à connotation négative sur le métier(2). Dans ce film, une comédie, l'interprète japonaise fait rire par son incompétence et sa duplicité : voilà une image à proscrire pour un métier qui demande un niveau d'expertise et de professionnalisme particulièrement élevé. Mais il ne faut peut-être pas crier au scandale dès que la caricature tourne au grotesque. Quand on a une histoire à raconter, si le personnage est secondaire à l'intrigue, on n'a généralement pas le temps d'entrer dans les détails et le septième art recourt inévitablement à des stéréotypes pour camper rapidement des personnages crédibles, faire avancer le scénario et retenir l'attention du spectateur.

Alors, que voit-on sur les écrans? Si vous voulez lire une étude approfondie sur le sujet, je vous conseille Translation goes to the Movies(3). En attendant, cet article se propose de survoler quelques films à succès qui font référence, directement ou indirectement, aux traducteurs et à la traduction.

(1) En utilisant ces termes, par flemme totale et assumée, j'inclus traducteurs et interprètes.

(2) Quelques actualités trouvées sur le web au 13/05/2014:

A farewell to arms? When technology gets lost in translation (The Independent)

Shared values that are lost in translation (Financial Times)

Gareth Evans: When words get lost in translation (WalesOnline)

(3) de Michael Cronin, Routledge, 2009. 


\section{Les super-héros de l'ONU}

L'Interprète(4) de Sydney Pollack (2005) est le seul film à gros budget, pour ne pas dire hollywoodien, que j'ai trouvé qui ait pour personnage principal un professionnel de la traduction. Dans ce film Nicole Kidman joue le rôle d'une interprète à l'Organisation des Nations Unies (ONU), à New York, qui découvre un complot visant à assassiner le président de son pays natal (pays fictif), la République de Matobo. À son tour traquée par les tueurs, elle est placée sous la protection d'un agent du FBI joué par Sean Penn. Le film se transforme ensuite en course contre la montre pour déjouer l'assassinat.

À première vue, la vie d'une interprète aux Nations Unies n'est pas ennuyeuse : il y a des attentats, des explosions, des snipers, des courses poursuites... En termes d'image donnée sur les traducteurs, le registre se place d'emblée dans l'héroïque puisque le personnage de Nicole Kidman évolue dans une histoire exceptionnelle, pleine d'événements exceptionnels. Grande et belle femme, de 35 à 40 ans, elle s'habille sobrement, toujours en pantalon, et privilégie les vêtements de couleur sombre. Studieuse, elle porte des lunettes quand elle travaille. Elle est célibataire et indépendante. Elle a une sensibilité artistique : elle est musicienne et joue de la flûte en amateur. Elle a grandi à l'étranger, dans un pays africain, et se montre émotionnellement fidèle à ses racines. Professionnelle, elle ne laisse pas ses convictions personnelles influencer son travail, même lorsque les pressions sont fortes. Mais elle n'est pas jugée digne de confiance par les agents du FBI qui la soupçonnent d'être une terroriste car elle est engagée politiquement et sait manier des armes. Enfin, elle est à l'aise avec l'informatique et communique avec son frère par email.

Le plus intéressant pour un spectateur issu des métiers de la traduction est, sans doute, qu'une bonne partie du film a été tournée au siège de l'ONU. Selon Wikipedia(5), source " plus-quefiable ", c'est le tout premier film à avoir obtenu l'autorisation de tourner à l'intérieur du bâtiment. Apparemment, cette autorisation a d'abord été refusée mais au dernier moment le Secrétaire général de l'époque, Kofi Annan, est intervenu pour tout arranger. M. Annan ne tarissait pas d'éloges sur le film :

[...] l'intention était de faire quelque chose de vraiment digne, quelque chose d'honnête et qui évoque le travail que fait cette organisation. Et c'est dans cet esprit que les producteurs et les réalisateurs ont abordé leur travail, et j'espère que vous allez tous convenir qu'ils ont réussi (6).

L'œil averti relèvera des détails intéressants sur les locaux de l'ONU : on voit des cabines d'interprétation à plusieurs reprises ainsi que la salle d'assemblée ; on nous explique comment

(4) Titre original The Interpreter.

(5) http://fr.wikipedia.org/wiki/L'Interprète.

(6) http://www.un.org/sg/offthecuff/?nid=719. 
la sécurité est organisée ; on nous montre les chambres fortes installées pour la protection des hauts responsables, etc. Le DVD(7) du film comprend, en outre, d'intéressants bonus qui présentent une compilation de ces images et d'interviews de "vrais " interprètes de l'ONU.

L'œil averti décèlera aussi un détail assez surprenant pour une production hollywoodienne : il n'y a pas de scène d'amour, même pas un baiser (il est bien connu que les interprètes mènent une vie de moine)! Malgré cet écart par rapport à la norme blockbustérienne, le film est un vrai polar, assez bien joué par Kidman et Penn et, quelques invraisemblances mises à part, c'est un bon divertissement. II donne certes une belle image du métier d'interprète et auprès d'un large public car, en termes de recettes, L'Interprète a fait un gros succès au box office. II traite néanmoins d'un personnage exceptionnel dans une situation exceptionnelle et on a du mal à imaginer que ce film influence particulièrement la perception du métier "de base "à cause de l'énorme écart entre le rêve (l'interprète à l'ONU qui déjoue un incident international) et la réalité de la situation de la plupart des artisans linguistes (traduction de documents techniques, interprétation lors d'une réunion, etc.).

\section{Pour les enfants (petits et grands)}

Dans un autre registre, les studios Pixar produisent des films gentils, intergénérationnels qui comptent parmi les plus grands succès de l'histoire du cinéma et qui, finalement, parlent assez souvent du langage, ce qui les conduit à évoquer indirectement les traducteurs et la traduction.

Le Monde de $\mathrm{Nemo}(8)$ est une sorte de road movie sous l'eau ayant pour personnages principaux des poissons. Nemo, jeune poisson clown, a été capturé par un dentiste australien qui voulait d'abord le mettre dans son aquarium personnel mais finit par l'offrir à sa nièce, la redoutable Darla. Marin, le père de Nemo, s'élance à sa recherche. Toutefois, comme c'est la première fois qu'il quitte son anémone, il a du mal à trouver son chemin et est un peu perdu dans la grande bleue. Heureusement il rencontre Dory, un poisson-chirurgien qui connaît l'anglais, la langue des signes, et sait parler baleine. Dory est pour ainsi dire guide-interprète !

Comment ce personnage est-il présenté ? Dory est de sexe féminin, décomplexée, sincère, mais un peu fofolle. Elle a une attitude très positive, optimiste, comme en témoigne sa chanson préférée :

(7) Studio Canal, 2005.

(8) Coproduit par Walt Disney Pictures et coréalisé par Andrew Stanton et Lee Unkrich, 2003. 
Nage droit d'vant toi, nage droit d'vant toi, nage droit d'vant toi d'vant toi d'vant toi ! Que faisons-nous, nous naaaaageons ! Nageons! J'adore la natatioooooon !(9)

Sa facilité à communiquer permet à Marin de mener à bien son projet. Conciliatrice, elle réussit à gérer des situations de conflit. Elle est amnésique et oublie aussitôt ce qu'on lui dit(10). Quand elle s'adresse à une baleine, elle continue à parler sa langue habituelle mais d'une voix grave et en prolongeant les voyelles, ce qui n'est pas forcément une bonne stratégie de communication interculturelle à conseiller aux enfants (et à leurs parents)(11)... Mais le bilan est globalement positif car Dory et ses compétences linguistiques permettent à l'histoire d'évoluer dans le bon sens.

La traduction automatique pointe son nez dans Là-haut(12) avec une meute composée de diverses races de chiens qui communiquent avec leur maître, et les autres humains de l'histoire, par l'intermédiaire d'un collier traducteur. D'ailleurs - et cela confortera sans doute les a priori négatifs des traducteurs humains - ce dispositif a été inventé par le " méchant " de l'histoire, un explorateur déshonoré qui n'a que des mauvais desseins. Autre ressemblance avec la réalité de la traduction automatique, il arrive que les colliers traducteurs se dérèglent et produisent des effets comiques! Détail intéressant et assez bien vu de la part des scénaristes, les colliers ne traduisent pas les aboiements des chiens mais leurs pensées, ce qui souligne la différence entre les mots et le message et le fait que la traduction ne consiste pas uniquement à remplacer les mots d'une langue par ceux d'une autre. Malheureusement le film véhicule aussi le message que la technologie réussit où l'homme échoue (aucun humain du film ne parle "le chien "), ce qui rappelle l'intox d'un certain géant de la recherche sur internet à propos de son service de traduction automatique.

Pour finir avec Pixar, le héros-jouet de Toy Story 3(13), Buzz l'Éclair, ranger de l'espace, se trouve pendant un certain temps en mode " espagnol ", ce qui non seulement le fait parler la langue ibérique mais aussi se comporter comme un toréador qui danse le flamenco (et tente de séduire Jessie, la cowgirl), démontrant ainsi - certes de manière absurde - le lien indéfectible entre langue et culture.

(9) Idem.

(10) Une interprète expérimentée me disait un jour qu'elle ne faisait plus que de la simultanée car sa mémoire défaillante ne lui permettait plus de faire de la consécutive.

(11) J'ai connu un marin écossais qui, à défaut de parler français, parlait anglais avec un accent français, ce qui semblait aider ses interlocuteurs parlant un peu anglais à le comprendre.

(12) Titre original Up, réalisé par Pete Docter et Bob Peterson.

(13) De Lee Unkrich, 2010, à noter que le titre a été traduit au Québec : Histoire de jouets 3 ! 


\section{Tous férus de technologie}

Le genre de la science-fiction est évidemment propice à créer des stéréotypes sur la traduction (ou son absence) car dès qu'on se met à voyager dans l'espace et à rencontrer des extraterrestres, on est évidemment en butte à des problèmes de communication. D'emblée, en l'absence de communication avec les extraterrestres, et donc de traduction, la seule interaction possible est la guerre, comme l'illustrent parfaitement La Guerre des mondes(14) ou Independence Day(15). Certains films ignorent complètement le problème, faisant parler tout le monde en anglais pour ne pas se perdre dans des explications compliquées qui prendraient trop de place (et trop de temps)(16). D'autres se reposent sur le concept du " traducteur universel „(17), dispositif assez improbable qui permet de faire avancer l'histoire sans trop se poser de questions.

Le plus célèbre de ces traducteurs universels est sans doute le robot C-3PO dans La Guerre des étoiles(18) qui maîtrise "plus de six millions de formes de communication ". Le robot est clairement masculin mais très peureux, pédant, un peu prude et la cible de beaucoup de farces. Il a dans la version américaine originale la voix d'un acteur britannique et une intonation snob qui lui confèrent la personnalité d'un professeur, ou d'un savant. II est amusant de noter que si le cinéaste se donne la peine de traiter le problème de la communication intergalactique en créant le personnage du robot-traducteur $\mathrm{C}-3 \mathrm{PO}$, ses héros sont généralement capables de comprendre les bips et sifflets des machines ainsi que les diverses langues des extraterrestres (qui rappellent des chants d'oiseau) sans aucune explication ni moyen d'aide apparent.

Avatar(19), film qui a enregistré les plus grosses recettes toutes catégories à ce jour, présente dans un premier temps une image assez négative du pouvoir de la traduction, qui est utilisée pour s'infiltrer parmi les habitants indigènes, obtenir leur confiance et mieux exploiter, par n'importe quel moyen, les ressources minérales de la planète Pandora. Tantôt la fiabilité des intermédiaires est mise en question par le peuple bleu, tantôt par les Terriens. Bien sûr le héros se rattrape à la fin mais les mauvaises intentions des mercenaires sont vaincues par le pouvoir de l'amour et non parce que la traduction permet à tous de se parler, de se comprendre et de trouver un terrain d'entente.

(14) Titre original War of the Worlds, Steven Spielberg, 2005.

(15) Roland Emmerich, 1996.

(16) Dans les séries télévisées Stargate SG-1 et Stargate Atlantis, la plupart des extraterrestres et des humains vivant sur d'autres planètes parlent l'anglais. Les réalisateurs de ces émissions ne cachent pas que cela leur permet d'éviter de passer dix minutes de chaque épisode à expliquer comment les personnages apprennent une nouvelle langue.

(17) http://en.wikipedia.org/wiki/Universal_translator

(18) Titre original Star Wars, George Lucas, 1977 pour le premier film.

(19) James Cameron, 2009. 
Une solution originale est présentée dans Le Guide du voyageur galactique(20). Malgré un contexte particulièrement poussé du point de vue technologique, c'est un poisson, nommé Babel, qui, une fois introduit dans l'oreille, permet au porteur de comprendre n'importe quelle langue. Dans cet univers, on suppose que les métiers de la traduction dépendront étroitement de la disponibilité d'une bio-ressource et non d'un savoir. Une source inépuisable et bon marché de ces poissons rendrait sans doute obsolète l'intervention d'un intermédiaire humain.

\section{Traduttore, traditore en haute mer}

Le regard porté sur les traducteurs par le célèbre réalisateur Alfred Hitchcock est sans doute plus proche de l'image qu'a eue l'espèce humaine de cette activité pendant la majeure partie de son histoire. OEuvre en noir et blanc de 1944, Lifeboat se passe, comme l'annonce le titre, sur un canot de survie au milieu de l'océan ; ses occupants sont les survivants du torpillage simultané d'un navire allié et d'un sous-marin allemand pendant la Seconde Guerre mondiale. À bord, on trouve plusieurs Américains et Britanniques et un Allemand, Willi, que les autres prennent pour un simple marin mais qui s'avère être le commandant de l'U-Boot qui a coulé leur navire. Sa présence divise les autres en deux camps : certains veulent le jeter à l'eau, d'autres insistent pour qu'il bénéficie du statut de prisonnier de guerre et soit gardé en vie.

Willi fait mine de ne pas parler l'anglais, langue qu'il maîtrise parfaitement, et la journaliste Connie Porter se porte volontaire pour faire office d'interprète, étant la seule anglophone à bord à parler allemand. De ces deux personnages, Willi est forcément le méchant car il est l'ennemi (Lifeboat est clairement un film de propagande) et Connie, étant une femme "moderne ", indépendante et sûre d'elle, met mal à l'aise les hommes forts du bateau qui cherchent à s'imposer en tant que leaders.

M. Hitchcock s'en donne à cœur joie pour exploiter la situation créée par les deux langues, suggérant une présence menaçante qui pèse sur les naufragés pendant tout le film. On se demande en permanence ce que l'un comprend, ce que se disent les deux principaux protagonistes, ce qu'on se raconte... Et, parmi les autres personnages, et chez le spectateur, ce questionnement sème le doute, génère une méfiance envers les traducteurs de fortune que le réalisateur met en scène.

(20) H2G2 : le guide du voyageur galactique, Garth Jennings, 2005, adapté de la mythique série radiophonique The Hitchhiker's Guide to the Galaxy de Douglas Adams (1978) et/ou du roman publié en 1979 (même titre, même auteur).

Pour la petite histoire, ce livre est dédié à ma marraine Claire Gorst et son désormais ex-mari Johnny Brock car, pendant la rédaction du livre, ils ont hébergé l'auteur Douglas Adams qui n'avait pas un rond (cf. http://ebookbrowsee.net/douglas-adams-the-hitchhiker-trilogy-pdf-d318888342). 


\section{Le noble métier}

Le portrait sympathique et extrêmement touchant de la traductrice allemande d'origine ukrainienne Svetlana Geier que peint Vadim Jendreyko dans son film documentaire La femme aux 5 éléphants(21), évoque parfaitement l'image qu'un traducteur passionné par son travail pourrait garder dans un coin de sa tête. Elle incarne le "noble métier ", ayant consacré sa vie à traduire des œuvres russes en allemand, notamment celles de Dostoïevski, qu'elle qualifie de "pachydermes ". C'est une femme brillante, douée, qui, à 21 ans, se voit accorder une bourse de l'Université de Humboldt malgré ses origines soviétiques. Elle est déterminée, débrouillarde, a vécu des grands événements de l'Histoire, survécu à la persécution en Ukraine(22) et à l'internement dans un camp pour travailleurs de l'Est à Dortmund. Elle est reconnue par ses pairs, considérée désormais en Allemagne comme la plus grande traductrice du russe. Érudite, inspiratrice, elle enseigne la traduction en faculté et est récipiendaire d'un doctorat honoris causa des Universités de Bâle et de Fribourg-en-Brisgau. Surtout, elle est en véritable symbiose avec son travail qu'elle vit en auteur et en artiste, comme en témoigne cet extrait célèbre :

Mon professeur disait toujours : "Il faut lever le nez quand on traduit ". Cela signifie qu'on ne traduit pas de gauche à droite, en suivant la langue, mais seulement après que l'on se soit approprié la phrase. Elle doit être digérée de l'intérieur, toucher le cour. Je lis le livre si souvent que les pages en sont trouées. D'abord je le connais par cœur. Ensuite vient un jour où enfin j'entends la mélodie du texte.

\section{Générique de fin}

Alors, après ce petit tour d'horizon cinématographique, qu'en pensez-vous ? Êtes-vous plutôt la dynamique super-héroïne Nicole Kidman, la patiente intellectuelle Svetlana Geier, Kapitän Willi l'opportuniste sous-marinier d'une moralité douteuse, l'efficace machine de traitement de texte C-3PO, ou bien Dory l'insouciant poisson bleu qui essaie, tant bien que mal, de nager droit devant lui ?

graham@mactrad.fr

Graham macLachlan est traducteur indépendant depuis 2004. Diplômé de l'Université Rennes 2, il est spécialisé dans la marine (sans parler d'une prédilection pour l'histoire). Outre son activité " pragmatique ", il peaufine actuellement sa traduction en anglais de La Main coupée de Blaise Cendrars. Un conseil : ne le branchez surtout pas sur les nœuds marins un jour où vous êtes pressé!

Il est président de la Société française des traducteurs depuis 2013.

(21) Nour Films, 2009.

(22) Svetlana Geier a travaillé comme interprète durant l'occupation allemande. Après la défaite de Stalingrad, la Wehrmacht se retire de Kiev et une grande partie de la population est déportée. Ceux qui restent sont menacés par de nouvelles épurations menées par les troupes de Staline.

Source : http://www.5elephants-lefilm.com/swetlana-geier 